\title{
MSC - a catalogue of physical multiple stars ${ }^{\star}$
}

\author{
A.A. Tokovinin ${ }^{1,2}$ \\ 1 Sternberg Astronomical Institute, Universitetsky prosp., 13, 119899 Moscow Russia \\ Internet: toko@sai.msu.su \\ ${ }^{2}$ Observatoire de Grenoble, Université Joseph Fourier, BP. 53, F-38041 Grenoble Cedex 9, France
}

Received May 9; accepted October 23, 1996

\begin{abstract}
The MSC catalogue contains data on 612 physical multiple stars of multiplicity 3 to 7 which are hierarchical with few exceptions. Orbital periods, angular separations and mass ratios are estimated for each subsystem. Orbital elements are given when available. The catalogue can be accessed through CDS (Strasbourg). Half of the systems are within $100 \mathrm{pc}$ from the Sun. The comparison of the periods of close and wide sub-systems reveals that there is no preferred period ratio and all possible combinations of periods are found. The distribution of the logarithms of short periods is bimodal, probably due to observational selection. In $82 \%$ of triple stars the close subsystem is related to the primary of a wide pair. However, the analysis of mass ratio distribution gives some support to the idea that component masses are independently selected from the Salpeter mass function. Orbits of wide and close sub-systems are not always coplanar, although the corresponding orbital angular momentum vectors do show a weak tendency of alignment. Some observational programs based on the MSC are suggested.
\end{abstract}

Key words: binaries: general — catalog

\section{Introduction}

It is generally recognized that stars of multiplicity higher than 2 are promising objects of study for several reasons. First, they often provide comprehensive data on stellar masses and luminosities that can be confronted with stellar evolution predictions. Secondly, they seem to offer an insight into the formation mechanisms of binary systems as first noted by Batten (1973). Indeed, the number of parameters that characterize a multiple system is greater

$\overline{\text { Send offprint }}$ requests to: Tokovinin (Sternberg Institute address).

* Tables 2 and 3 are only available in electronic form at the CDS via anonymous ftp to cdsarc.u-strasbg.fr (130.79.128.5) or via http://cdsweb.u-strasbg.fr/Abstract.html than that of a simple binary, and in this multi-parameter space the information on the formation history may be obtained from period ratios, mass ratios, relative orientation of orbital planes, etc.

Surprisingly, no general catalogue of multiple systems has been published until now, although there are several "partial" catalogues with various limitations. Fekel (1981) published a list of triple systems with periods less then 100 years. On the other hand, a catalogue of long-period visual triple stars where all 3 components are resolved was provided by Anosova (1988). It was later extended by Popovic (1991) to include some 700 visual triple stars within $200 \mathrm{pc}$ from the Sun. Only a fraction of them can be considered as genuinely triple, i.e. with proved physical relation between all 3 components. The catalogue of nearby wide binary and multiple systems (Poveda et al. 1994) includes triple and quadruple systems which are physical, but, like the two previously cited catalogues, ignores all short-period sub-systems. Chambliss (1992) published a list of eclipsing binaries with visual companions.

The fragmentary nature of the existing information on multiple stars is due not only to the personal preferences of the authors, but also to the intrinsic difficulty of discovering multiple stars. The effects of observational selection are already very severe in case of binary stars and are still more restrictive for the systems of higher multiplicity. Often the discovery of such systems has resulted from accidental combination of different observing techniques. The distribution of orbital periods of binary stars is shown to be continuous in the range from 1 to $10^{9}$ days (Duquennoy \& Mayor 1991). If we are to understand the statistics and origin of multiple stars, it is evident that the information throughout the full period range is needed. Hence, there is a need of the general catalogue of multiple stars that would combine the results obtained by all observational techniques, without additional restrictions other than those imposed by the techniques themselves. The compilation of such multiple star catalogue (hereafter MSC) is the aim of this study. 
In Sect. 2 the input sources of MSC are reviewed and general remarks on the definition and description of multiple stellar systems are given. The procedures used to estimate and code the parameters of sub-systems are given in Sect. 3, while the catalogue itself is described in Sect. 4. Such properties of the MSC as completeness, period and mass ratio distribution, orbit coplanarity are studied in Sect. 5. The conclusions and future research programs related to MSC are given in Sect. 6 .

\section{General remarks}

\subsection{Input sources}

Fortunately, the investigators of binary stars were generally very attentive to multiplicity higher than 2 and provided corresponding comments. These comments were amply used in the selection of objects for MSC. In particular, we scanned the comments to the Washington double star catalogue (Worley \& Douglas 1984, hereafter WDS), to the Fourth catalogue of orbital elements of visual binary stars (Worley \& Heintz 1983, hereafter VBO) and the Eighth catalogue of spectroscopic orbits (Batten et al. 1989, hereafter SB). These two latter catalogues were also intercompared directly to find objects in common.

The Third preliminary version of the Catalogue of nearby stars (Gliese \& Jahreiss 1991) was scanned to find multiple systems and this selection was checked later with the catalogue of Poveda et al. (1994) based on the same source. Notes to the Fifth edition of the Yale Bright star catalogue (Hoffleit \& Warren 1991) were used as well for initial selection of objects. Multiple stars mentioned in the Second catalogue of speckle interferometric measurements of binary stars (McAlister \& Hartkopf 1988) were added.

Naturally, the multiple systems described in the current literature or studied by the author were also included. In 1994 a program of radial velocity measurements of late-type components of multiple systems with correlation radial velocity spectrometer was started, and its results (tests of physical relation between visual components and discoveries of new close sub-systems) are incorporated into MSC.

Finally, the multiple star lists mentioned in the Introduction were merged into MSC. From the catalogue of Popovic (1991) we took only systems which he considered to be physical. Popovic used the WDS as input source, but this latter in fact contains many more visual multiples. Dommanget (1988) gives the number of visual multiples in CCDM (which contains approximately the same objects as WDS) as 5800. The physical relation between the components of these systems is rarely proved, so it seemed reasonable to restrict the present study to the list of Popovic until the nature of the remaining systems is elucidated, e.g. by the HIPPARCOS observations.

It can be stated that MSC is "complete" in the sense of taking into account most of available sources: any known multiple system may be found in MSC with a good probability, with exception of visual multiples as explained above.

The available data on multiple stars are very inhomogeneous in nature and quality, and, consequently, no uniform and coherent description of all known multiple systems is possible. Our approach is to give a reasonably complete set of parameters for each system, bearing in mind that the reliability and quality of these data is very different, ranging from crude and uncertain estimates to good measurements. Answering the potential criticism, we feel that it is actually the only possible way to combine the information, and a necessary step towards the definition of future observing programs that would provide data of better quality and homogeneity.

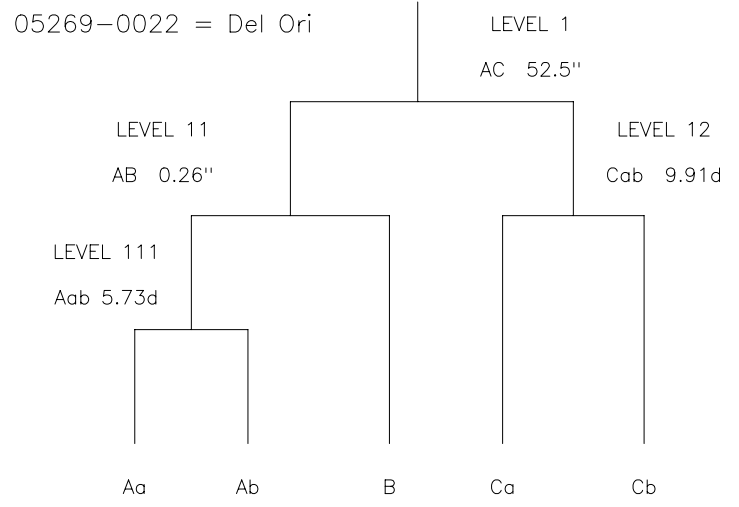

Fig. 1. Mobile diagram of the quintuple system $\delta$ Ori and the corresponding level codes. Visual components are designated by upper-case letters, spectroscopic components by second small-case letters. Separations are given for visual systems, periods for spectroscopic systems

\subsection{Spurious multiple stars}

Our aim is a catalogue of physical systems containing at least 3 stars. In case of wide visual double stars it must be proven that their components are not optical. The criteria used to identify optical components are detailed in Sect. 3.1. Wide systems with uncertain status were not included in our final list, but were retained in the database for further observations. It turned out that a large fraction of systems listed as physical by Popovic (1991) contains optical companions.

As for the close spectroscopic sub-systems, their reality is not always unquestionable, too. In the past radial velocity observations of poor quality or with different systematic offsets has led to the identification of spectroscopic binaries which are in fact single. A good example is Gl 4AB, both components of which are noted as spectroscopic binaries in the old catalogue of nearby stars (Gliese 1969) but have been found to have constant radial velocity 
(Tokovinin 1992). So, the stars without computed spectroscopic orbits are considered as spectroscopic binaries only when the velocity variability is confirmed by modern observations.

We caution the potential users of MSC that a few spurious multiple systems may still be listed despite the efforts to delete them.

\subsection{The long period limit}

Several stars with common distance and common spatial motion do not necessarily form a multiple stellar system. They may be just members of a stellar cluster, association or moving cluster. Is there a formal distinction between multiple stars and clusters? The only significant difference concerns the type of motions, quasi-keplerian for hierarchical multiple stars and stochastic for other stellar groups. This criterion is however both questionable and impractical. The possibility of quasi-periodic motions is easier to check. It is known that wide pairs with separations greater than $\approx 10^{4}$ A.U. are disrupted by encounters with stars and molecular clouds (e.g. Weinberg \& Wasserman 1988), i.e. are not stable binaries. This consideration lead Abt (1988) to propose a distinction between wide binary stars and co-moving pairs. It seems thus inevitable to impose an upper limit on the separations of multiple star components in order to distinguish them from other stellar groups. The statistical difference between multiple stars and open clusters was discussed by Dommanget (1977).

An interesting conclusion was reached by Larson (1995) from a study of the clustering properties of young pre-main sequence stars. He found that for separations $r$ greater than $0.04 \mathrm{pc}$ the probability of finding a companion is represented by a power law which is related to the fractal structure of star-forming clouds. For smaller separations this probability is proportional to $r^{-2}$ as typical for multiple stars. This finding strengthens the idea of limiting component separation that distinguishes multiple stars from clusters and associations.

The "Larson limit" is of the same order as the stability limit and corresponds to an orbital period of $\approx 10^{6}$ years for 1 solar mass system. This period was used in MSC compilation as a guideline for rejection of wide pairs. However, no strong limit was imposed and some systems with longer periods were still included. It must be kept in mind that the separation between the members of the Trapezium system is less than the limit quoted above, and still this system is just the dense core of a stellar cluster. Until the relation between multiple systems and clusters becomes clearer, it is safe to adopt a somewhat relaxed long period limit and to keep in MSC non-hierarchical systems.

\subsection{Description of hierarchical systems and component census}

The majority of multiple systems are hierarchical and so can be considered as a combination of binary sub-systems. Evans (1968) proposed to describe hierarchical multiple systems by mobile diagrams. The widest system forms the upper level of the diagram, any component that is a closer double is represented as a bifurcation at next level, etc. Such diagram is simply a binary tree. We choose to encode the place of each sub-system in a mobile diagram by an integer number called level. The widest system is designated level 1, the sub-system of its primary component is level 11, and that of the secondary is level 12. If there are still closer sub-systems, they will be designated levels $111,112,121$ or 122 , and so on. This is evidently not a very efficient way to encode a binary tree, but it is easy to understand and to decode. The mobile diagram and the associated levels are illustrated in Fig. 1. Maximum number of levels found in MSC is 4, e.g. from 1 to 1111.

Level designations can be generalized to include nonhierarchical systems, called also trapezia, which contain more than two components with comparable separations. The separation ratio $3: 1$ is generally used to distinguish trapezia from apparently hierarchical systems. For example, a trapezium system with 3 visual components A, B, C will be represented as two binaries $\mathrm{AB}$ and $\mathrm{AC}$, but these "binaries" will have the same level (level 1). Should it happen that components $\mathrm{B}$ or $\mathrm{C}$ are in turn spectroscopic binaries, they would be designated levels 12 or 13 .

The attribution of a sub-system to the particular hierarchical level may change with the discovery of new components. The multiple systems with complete component census (complete systems where the existence of undiscovered companions is excluded by observations and stability considerations) are exceptionally rare, if not inexistent. Hence, the terms "triple", "quadruple", etc. reflect the current observing status rather than the true multiplicity. The definition of a sample of complete multiple systems is of great interest, and MSC may be used as a source of observing programs needed to construct such sample.

\section{The contents of MSC}

\subsection{Sub-system types and component designations}

Each sub-system is associated with a particular observing technique (or their combination) used to discover and study it, which hereafter is called type. In MSC it is coded as a sequence of letters. Their meaning is explained below.

Wide physical double stars are coded by the letter $\mathbf{C}$ (for C.P.M. - common proper motion pairs). It is followed by one or several small letters describing what criteria were used in testing the physical relation of the components: 
- m: proper motions of components are known and are equal within their errors which are assumed to be $\pm 00^{\prime \prime} 015$.

- h: hypothetical dynamical parallax as computed from the relative component motion does not exceed by more than 3 times the photometric parallax. Systems with fixed relative position of components also belong to this class. The hypothetical parallax $h=$ $0.418\left(\rho \mu^{2}\right)^{1 / 3}$, where $\rho$ is the apparent separation of a binary and $\mu$ is the relative motion of components, is on the average proportional to $p M^{-1 / 3}$, where $p$ is parallax and $M$ is mass sum in solar units (Russel \& Moore 1940). Relative motion $\mu$ was computed form the first and the last observations given in WDS (admittedly a risky procedure because of the insufficient accuracy of the observations). This criterion has only a statistical sense, but it proved to be very useful in rejecting optical companions.

- r: radial velocities of components are equal within measurement errors.

- p: component distances, as estimated from spectral types and apparent magnitudes, are mutually consistent.

When neither of these criteria is verified the system is designated by $\mathbf{c}$. In some cases there is also a question mark which means a likely optical system or a contradiction between different criteria. Uncertain wide systems are included only if one of their components is at least triple.

When components are resolved and are separated by less than 3 " the system is called "visual" and is designated by $\mathbf{v}$. Interferometrically resolved binaries are also included in this category. It is assumed on statistical grounds that all close visual systems are physical. In some cases it can be proved in the same way as for wider systems, e.g. by hypothetical parallax. Visual binaries with computed orbits are designated by $\mathbf{V}$. Binary stars discovered by lunar occultation technique are coded by $\mathbf{o}$.

When the binary nature is deduced by astrometric techniques (i.e. by deviations from linear motion on the sky or from the keplerian motion of components in a wider sub-system) the letter $\mathbf{a}$ is used, or $\mathbf{A}$ if an astrometric orbit is computed.

Binarity discovered from velocity variations or from composite spectral type is coded as $\mathbf{s}$ (or as $\mathbf{s} \mathbf{2}$ if double lines were noted). Spectroscopic binary stars with computed orbits have designations S1 (single-lined) or S2 (double-lined).

Eclipsing binary stars have code $\mathbf{E}$ if orbital period is known, code e otherwise. Code $\mathbf{E}^{*}$ means a sub-system discovered by eclipse timing.

The traditional identification of visual components by upper-case letters A, B, etc. as given in WDS was kept in MSC, to avoid component confusion as much as possible. The additional visual components are designated by letters P, Q, etc. as usual. Spectroscopic sub-systems are identified by appending lower-case letters, e.g. Aa, Ba. A "component" may turn into "sub-system" if a new close component is discovered. However, the sub-systems already known would not be modified by such a discovery, only their level might change.

\subsection{Mass estimates}

The masses of individual components or sub-systems in solar mass units are given in the MSC. Mass codes describe the method used for the estimation:

- s sum of masses of lower-level sub-systems that constitute a "component" of a higher-level binary.

- * masses are given in the original publication. They are usually obtained either from an orbital solution or from a model of the system that fits component's colors and spectral types.

- a mass is estimated from the spectral type using the data from Allen (1973). This procedure works well for main-sequence stars, but mass estimates for luminosity classes III, II, I are very uncertain, because there is no unique relation between spectral type and mass. In some cases only the color index $B-V$ is known, and the mass is estimated under the assumption of luminosity class V. Stars of the type Am are assumed to have mass of $2.5 M_{\odot}$, mass of white dwarfs is assumed to be $1 M_{\odot}$.

- q secondary mass estimated from primary mass and mass ratio of double-lined spectroscopic binary.

- m minimum secondary mass for single-lined spectroscopic binary.

- $\mathbf{v}$ mass estimated from magnitude difference of a visual binary under the assumption that both components are main-sequence stars, and with the data of Allen (1973).

- : same as above when the magnitude difference is not known and is assumed to be $2^{\mathrm{m}}$, e.g. for interferometric binaries.

The order in which the items are listed reflects the priority of different mass estimation procedures which are chosen automatically according to the available information and the type of system. Evidently, the quality of mass estimates given in MSC is very different, but the mass code indicates the quality without ambiguity.

\subsection{Distance estimates}

The methods of distance estimation and the corresponding codes are listed below in order of preference:

- ORB: orbital parallax determined from a combined visual-spectroscopic orbit.

- DYN: dynamical parallax calculated from the elements of a visual orbit and the sum of masses estimated as described above. If masses are not known, a mass sum of $2 M_{\odot}$ is assumed.

- GL: trigonometric parallax from Gliese \& Jahreiss (1991). 
- p: spectroscopic parallax estimated from spectral type and apparent magnitude. Data from Allen (1973) are again used. No reddening or absorption is taken into account. The number(s) after letter $\mathbf{p}$ indicate which of the visual components was taken, e.g. p12 means that both primary and secondary have similar spectroscopic parallaxes. Parallaxes estimated from mainsequence components were preferred when possible because spectroscopic parallaxes of giants and supergiants may be very imprecise.

- bib: distance estimates taken from literature, e.g. inferred from cluster membership.

\subsection{Periods and separations}

The decimal logarithm of orbital period in days is given for each sub-system. For sub-systems with known orbits (types V, A, S1, S2, E) it is the true orbital period, otherwise it is estimated from component separation, distance to the system and mass sum. It is assumed that the observed component separation $\rho$ is equal to the semi-major axis of the orbit $a$ (the statistical studies show that the average factor relating $a$ to $\rho$ is indeed close to unity). These estimates are useful for statistical purposes and as an indication of the possibility of orbit computation.

The meaning of the angular separation depends on system type. For systems with known visual orbit it is equal to $a$. For visual and C.P.M. systems $\rho$ is given (if it changed with time, the first observation is usually given). For occultation binaries the projected separation is given. Finally, for spectroscopic and eclipsing systems $a$ is calculated by the third Kepler law. The position angles are also given. They can be helpful for identification of wide components but practically meaningless for close binaries in orbital motion.

The summary of system types and the corresponding meaning of period and separation is given in the Table 1 , where $P$ is the true orbital period, $a$ is the apparent semi-major axis, $p$ is the parallax and $M$ is the mass sum.

\subsection{Magnitudes and spectral types}

For each sub-system the visual magnitudes and spectral types of the components are given. These were collected from all available sources and are of very unequal quality. Evidently, MSC is not a photometric catalogue! However, the information on magnitudes and spectral types is essential in understanding the nature of the systems and in planning of observations.

Mutual inconsistency of component and system magnitudes is frequent in MSC because no special effort was made to adjust data from different sources. The distinction between individual and combined magnitudes is not always clear, although individual magnitudes were preferred for "single" components and the combined magnitudes for unresolved multiple components. In some instances the in- dividual component magnitudes were calculated from the combined magnitudes and the published magnitude difference. For some systems the yet unpublished area-scanner photometry is given (Shatskii 1996). If the spectral type in not known, e.g. for faint visual components, the $B-V$ color is given instead when available.

\section{Catalogue description}

The catalogue consists of Table 2 (main data) and Table 3 (cross-index) which are available in electronic form at CDS (Strasbourg). Data on one multiple system are given as an example in Table 4 in the same format as in Table 2.

The form of Table 2 is the result of the compromise between two conflicting requirements. First, it seems natural to group various data on any given system in the same place to facilitate their retrieval, evaluation and comparison. On the other hand, the ASCII tabular file with fixedformat items is desirable for computer analysis. Table 2 is an ASCII file with lines of different types. Each line begins with the MSC code of the system based on the 1900.0 equatorial coordinates of the primary component. The MSC code is similar, but not equivalent to IDS (e.g. + and - signs are used instead of letters $N$ and $S$ ). The second item of each line represents the key that defines the type of line and hence the information it contains. Systems are separated by blank lines. Using Unix command sort, it is easy to extract lines of the same type to a fixed format tabular file. Still the data on a system are not separated and can be searched and viewed by means of text editor or extracted by grep command if the MSC code is known. The MSC code can be obtained from other identifiers, such as HD or ADS, with the help of the cross-index file (Table 3).

The unknown parameters are given zero value. This may cause some inconvenience or mistakes. On the other hand, there are no missing items, and all lines of given type always contain equal number of items.

The identification line is the first line for a system (code $\mathbf{A}$ ) and contains common identifiers: HD number, $\mathrm{DM}$ number (BD, CD or CPD), HR number, ADS number (Aitken 1932), and other names if available (Flamsteed name, Gliese catalogue number Gl or GJ, variable star name, etc.). Zero means that the object is not found in a corresponding catalogue. Only one HD, HR or DM number corresponding to the primary is given for a system. The spaces in each catalogue identifier are converted to underline symbols.

The distance line (identified by the code $\mathbf{D}$ ) contains additional information common to the whole multiple system. The equatorial coordinates for 2000.0 of the primary are given in the form of real numbers: hh.mmss and $d d .{ }^{\prime \prime \prime} "$ ". Then the proper motions in R.A. and declination in milliarcseconds per year are given. They were collected from various sources. Some proper motions were 
Table 1. Codes of system type and meaning of period and separation

\begin{tabular}{llll}
\hline Code & Description & Period & Separation \\
\hline$C$ & physical wide binary, $\rho>3^{\prime \prime}$ & $(\rho / p)^{3 / 2} M^{1 / 2}$ & $\rho$ \\
$v$ & visual or interferometric binary & $(\rho / p)^{3 / 2} M^{1 / 2}$ & $\rho$ \\
$V$ & visual binary with computed orbit & $P$ & $a$ \\
$A$ & astrometric binary & $P$ & $p P^{2 / 3} M^{1 / 3}$ \\
$o$ & occultation binary & $\left(\rho^{*} / p\right)^{3 / 2} M^{1 / 2}$ & projected $\rho^{*}$ \\
$s, s 2$ & possible SB (var RV or composite) & - & - \\
$S 1, S 2$ & single- or double-lined SB orbit & $P$ & $p P^{2 / 3} M^{1 / 3}$ \\
$e$ & eclipsing binary & - & - \\
$E, E *$ & eclipsing with known period & $P$ & $p P^{2 / 3} M^{1 / 3}$ \\
\hline
\end{tabular}

Table 4. MSC sample: data on one multiple system

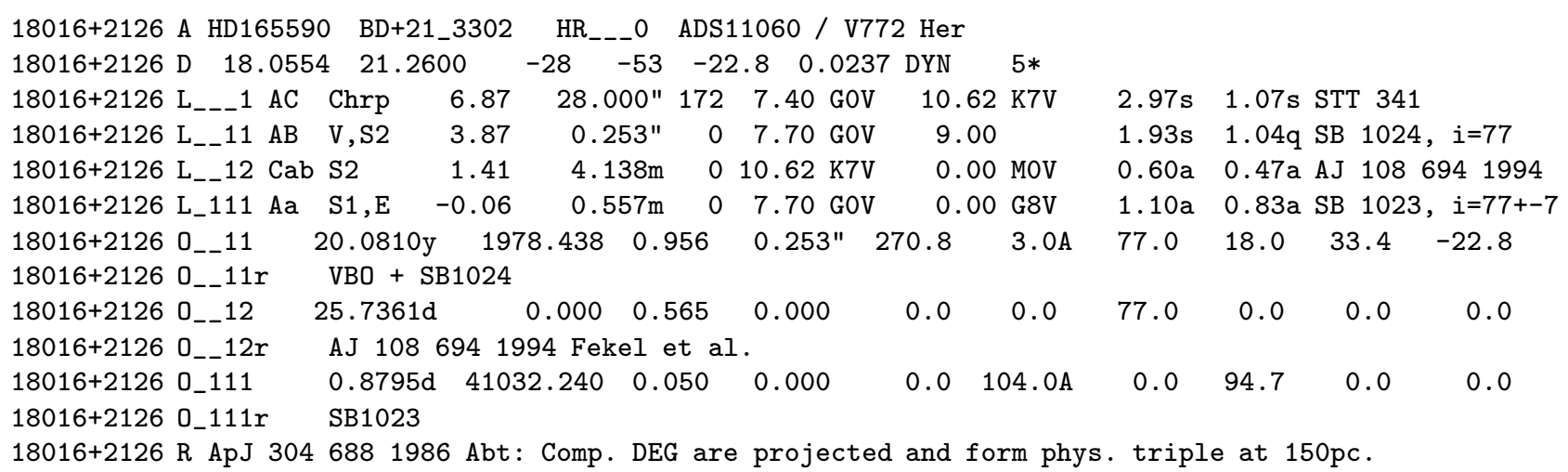

taken from the recent "4 million" catalogue (Volchkov et al. 1992). Radial velocity is also provided, followed by parallax and its code as detailed in Sect. 3.3. The last item is multiplicity, marked by asterisk and a letter " $\mathrm{T}$ " for trapezium systems.

Then follow the system lines, one per each sub-system, with codes beginning by $\mathbf{L}$. The numbers after $\mathrm{L}$ are the hierarchical level of this sub-system (Sect. 2.4). System lines contain the component designation, system type codes (Sect. 3.1), the decimal logarithm of the orbital period in days (or asterisk if period is not known), angular separation, its units (' for arcminutes, " for arcseconds and $m$ for milliarcseconds), position angle in degrees, visual magnitude and spectral type of the primary (or its $B-V$ color index if the spectral type is not known), visual magnitude and spectral type or color index of the secondary, masses of the primary and secondary components (in units of solar mass) with by their codes. The last 20 characters of system line are the remarks giving additional information either on the system (identification in WDS or SB catalogues, short reference) or on its secondary (HD, HR, DM numbers when available). In some cases the orbital inclination is also given in the remarks.
When visual or spectroscopic orbital elements are known, they are given on the two consecutive orbit lines which have codes beginning with $\mathbf{O}$ and containing the same 4-number level designations as the system lines. Visual and spectroscopic orbits are given in the same format; in some cases they were combined together as indicated by + sign in the remarks. The orbital elements are given on the first orbit line in the following order: period, epoch of periastron passage, eccentricity, semi-major axis with its units (" for arcseconds and $m$ for milliarcseconds), PA of ascending node, longitude of periastron followed by letter A or B specifying the component to which the last two parameters refer, orbital inclination, semiamplitudes of the primary and secondary, center of mass velocity in $\mathrm{km} / \mathrm{s}$. Period and periastron epoch are given either in years or in days (in this latter case 2400000 must be added to the epoch). The second orbit line has letter $\mathbf{r}$ appended to the code and contains remark that provides either reference to catalogues of spectroscopic (SB) and visual (VBO) orbits or bibliographic reference, given as journal abbreviation, volume, page, year and sometimes author name. Codes T94 or T95 denote my unpublished orbits. 
The optional remark lines are coded by $\mathbf{R}$ and contain text in free format, mostly additional references.

Table 3 is a cross-index file that is provided for convenience. It has a fixed format. Each line contains MSC code of the multiple system, component identification letter and HD, DM, HR, ADS numbers. Missing numbers or items are replaced by asterisks. A multiple system may have several lines in Table 3 if one of its visual components has separate identifiers.

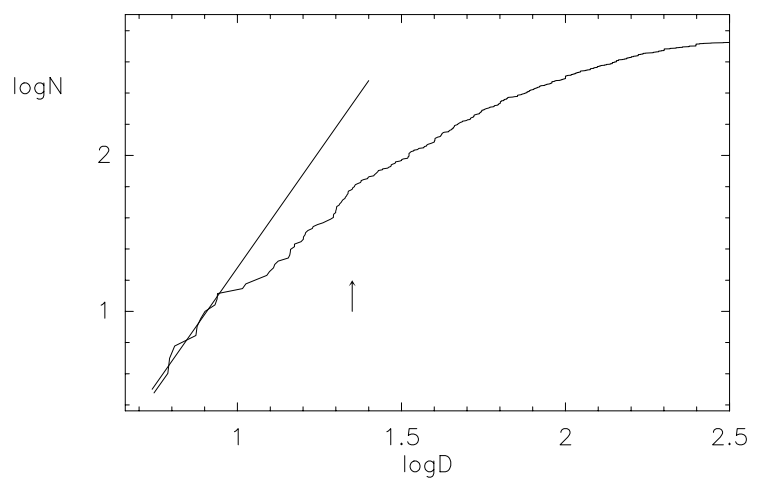

Fig. 2. Number of multiple systems $N$ within distance $d$ in the MSC. The expected $d^{3}$ proportionality is indicated by the straight line. Distance limit of the Catalogue of nearby stars is shown by the arrow

\section{Some properties of the MSC}

\subsection{Completeness}

A usual way to check the completeness of any catalogue is to verify if the number of objects $N(d)$ closer than a given distance $d$ does indeed increase as $d^{3}$. The $N(d)$ for MSC is given in Fig. 2. The departure from $d^{3}$ law begins already at the distance of $6 \mathrm{pc}$. The straight line is an attempt to fit the first points by the cubic law. It predicts the number of multiple systems within $10 \mathrm{pc}$ to be 19. The total number of stars within $10 \mathrm{pc}$ being 236 (Gliese \& Jahreiss 1991), the fraction of multiple systems can be estimated as $6 \%$. Thirteen systems within $10 \mathrm{pc}$ with mean primary mass of $0.66 M_{\odot}$ are actually listed in MSC.

The limit of the Catalogue of nearby stars is shown by the arrow in Fig. 2. The comparison of actually listed (62) and predicted (217) numbers of multiple systems within $22.5 \mathrm{pc}$ shows that only about $1 / 3$ of them are discovered. If the primary's mass dependence on distance is examined, it becomes evident that multiple systems with primary mass less than $0.5 M_{\odot}$ are all concentrated within $30 \mathrm{pc}$, and the lower mass limit increases gradually with the distance.

The little that was said on the MSC incompleteness suffice to affirm that the "population" of MSC must not be identified with the real population of multiple stars, and any statistical inferences from the MSC must be taken with caution. For example, the relative proportion of different multiplicities in MSC $(3: 4: 5: 6: 7=470$ : $108: 27: 5: 2)$ is not necessarily representative of the true proportion, because the systems are not complete (cf. Sect. 2.4). The following sub-sections describe the statistical properties of the catalogue rather than the statistics of multiple stars, because no allowance for observational selection was made.

\subsection{Period distribution}

In Fig. 3 the period of wide sub-system $P_{\mathrm{L}}$ is plotted in a logarithmic scale against the period of the corresponding close sub-system $P_{\mathrm{S}}$ at the adjacent hierarchical levels. A system of multiplicity higher than 3 provides more than one point in this figure. The line marks equal periods. The absence of systems near this line clearly indicates that stability criteria are satisfied: for the majority of multiple systems $P_{\mathrm{L}}>10 P_{\mathrm{S}}$. The smaller $P_{\mathrm{L}} / P_{\mathrm{S}}$ ratios exist, but they correspond mostly to visual multiples and are likely to be the result of uncertain period estimates from projected separations.

The upper limit on $P_{\mathrm{L}}$ was discussed above (Sect. 2.3). On the other hand, there are no $P_{\mathrm{S}}$ shorter than $0.3 \mathrm{~d}$, a typical period of main sequence contact binary. It can be noted that multiple systems in the $\log P_{\mathrm{S}}-\log P_{\mathrm{L}}$ plane occupy almost all space in the triangle defined by these limits. Apart from this, no evident relation between $P_{\mathrm{S}}$ and $P_{\mathrm{L}}$ can be seen. In particular, the period ratio $P_{\mathrm{L}} / P_{\mathrm{S}}$ (its logarithm is the distance of a point above the limiting line $P_{\mathrm{L}}=P_{\mathrm{S}}$ ) does not appear to have any preferential value. This conclusion seems to be immune to selection effects and it must be kept in mind in attempts to understand the formation mechanisms of multiple systems.

The distribution of points in the limiting triangle is not uniform. In Fig. 4 the distribution of $\log P_{\mathrm{S}}$ is plotted. It shows a marked depression for $0.7<\log P_{\mathrm{S}}<4$. For comparison, the period distribution in the $\mathrm{SB}$ catalogue is also plotted. It is clear that severe selection effects are influencing the shape of $\log P_{\mathrm{S}}$ distribution for $P_{\mathrm{S}}>5 \mathrm{~d}$. The same explanation may be valid for the apparent maximum at $P_{\mathrm{S}} \approx 30 \mathrm{y}$. At a typical distance of $30 \mathrm{pc}$ the corresponding semi-major axis (12 A.U. for $2 M_{\odot}$ mass sum) is equal to 0 "' 4 , suspiciously close to the resolution limit of visual discovery techniques. A speckle-interferometric survey of nearby stars could be particularly helpful, providing the order-of-magnitude increase in angular resolution needed to decouple the observational limit from possible real features in the period distribution.

The diagram $\log P_{\mathrm{L}}-\log P_{\mathrm{S}}$ for triple systems was discussed by Duquennoy \& Mayor (1986). They noted a bimodal distribution of $P_{\mathrm{S}}$ with a gap at $P_{\mathrm{S}} \approx 100 \mathrm{~d}$ and stated that this gap can hardly be explained by the discovery biases. Examining Figs. 3, 4, we do find the deficiency 


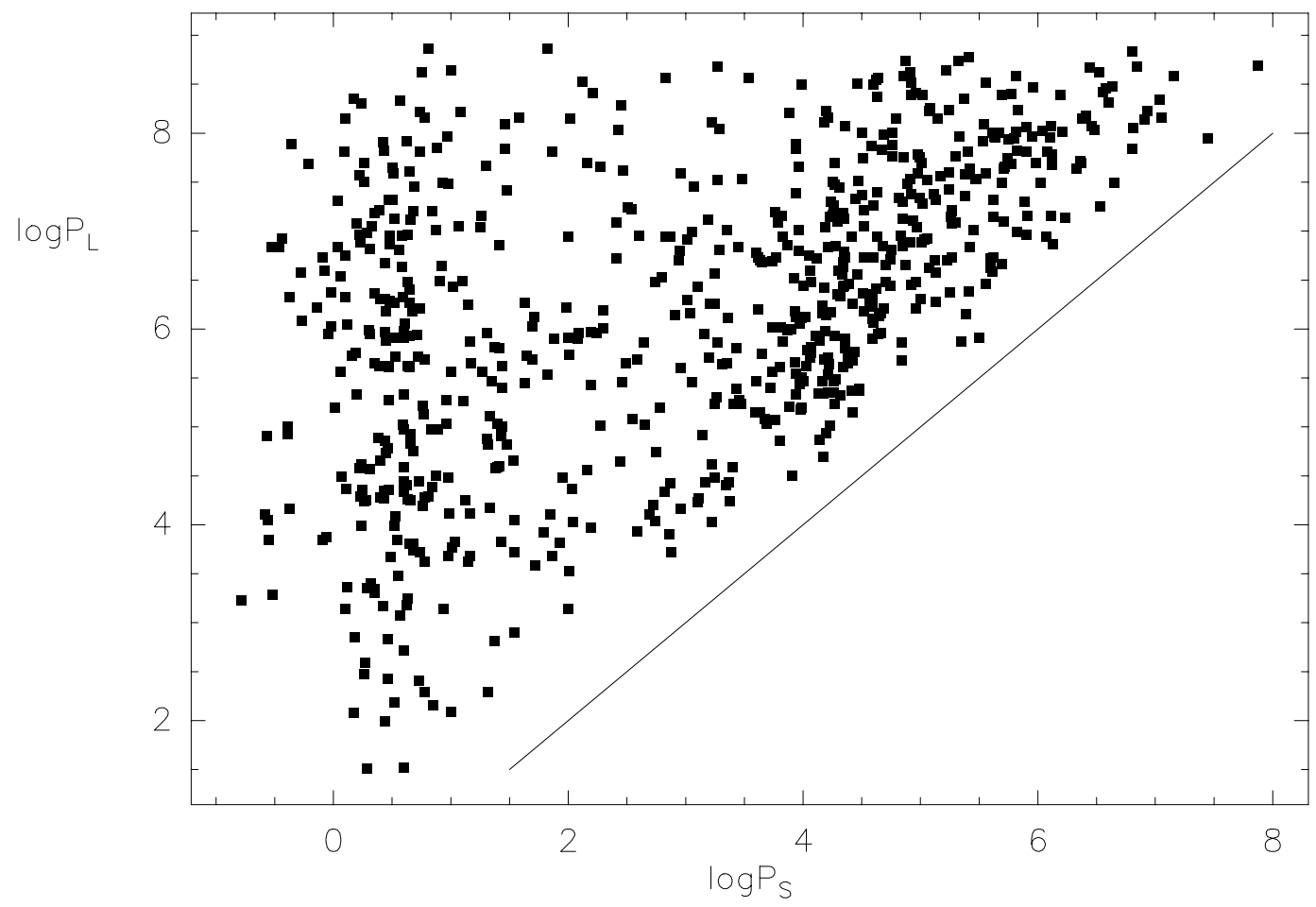

Fig. 3. The relation between the logarithms of the orbital periods of short- and long-period sub-systems $P_{\mathrm{L}}$ and $P_{\mathrm{S}}$ (in days) at adjacent hierarchical levels

of points at $\log P_{\mathrm{S}} \approx 2$. However, as mentioned above, it may still be related to discovery limitations.

There seems to be no dependence of $P_{\mathrm{L}}$ and $P_{\mathrm{L}} / P_{\mathrm{S}}$ on the mass of primary component. When only systems with unevolved primaries from 0.8 to $1.5 M_{\odot}$ are selected for the plot, the general aspect of Fig. 3 does not change.

The discussion of $P_{\mathrm{L}}-P_{\mathrm{S}}$ relation may be influenced by the fact that some components still remain undiscovered: the periods presently attributed to the adjacent levels of hierarchy may be actually separated by undiscovered intermediate levels.

\subsection{Level asymmetry and mass ratio distribution}

Even a cursory examination reveals that binary trees representing multiple systems are generally asymmetric. It is the primary that most often contains close sub-systems. Table 5 gives the number of different levels in MSC. Restricting ourselves to apparently triple systems, we find 381 cases of level 11 and 83 cases of level 12, i.e. $82 \%$ of the "triple" stars have the close sub-system associated with the primary. It is not clear to which extent this preference is due to selection effects (bright primary stars are usually better studied).

This level asymmetry is evidently related to the mass ratio distribution. Let us consider the parameter $Q$, the ratio of distant component mass to the mass of the pri-

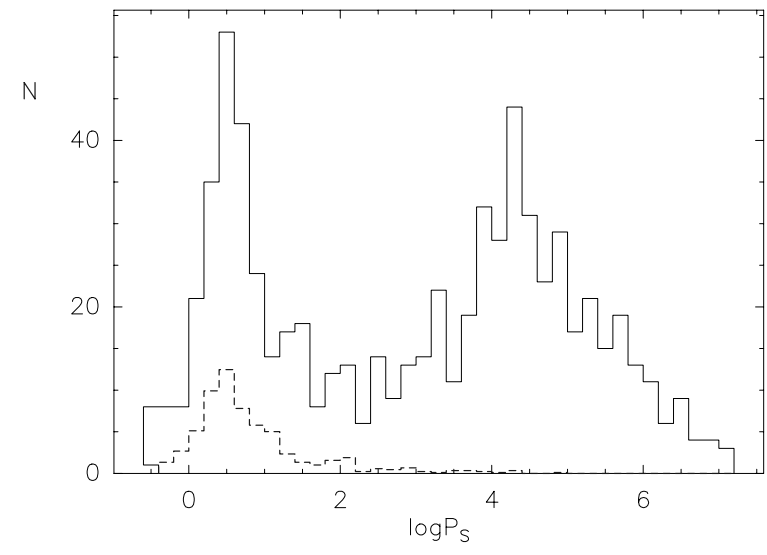

Fig. 4. The distribution of the logarithm of short periods plotted in Fig. 3. The scaled distribution of the periods of spectroscopic binaries from the catalogue of Batten et al. (1989) is plotted as dashed line for comparison. It is possible that the depression in the period range from 5 to $10^{4}$ days is due to observational selection

mary in a close sub-system. If the distant component is itself non-single, its mass sum is used in calculating $Q$, and such a quadruple system provides 2 values of $Q$, one for each of close sub-systems. The distribution of 702 values of $Q$ is given in Fig. 5 (full line). The small "step" at 


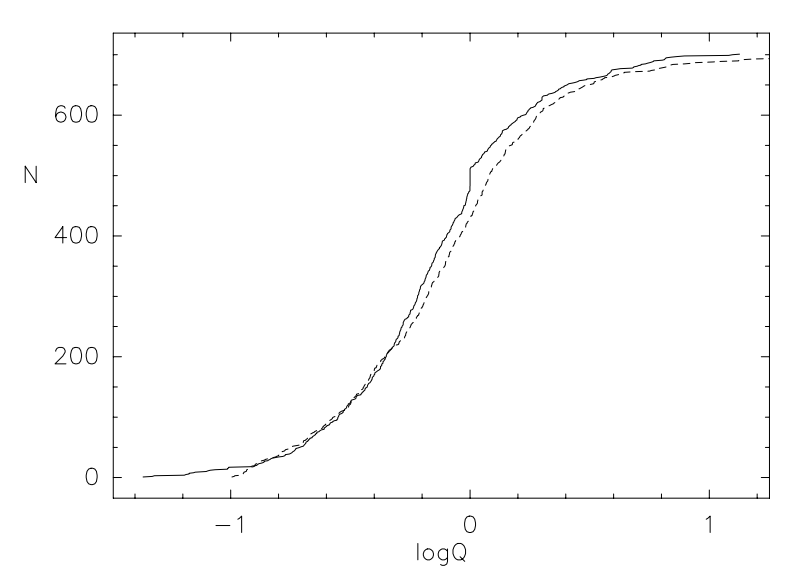

Fig. 5. Cumulative distribution of $Q$, the ratio of the distant component mass to the primary mass in a close sub-system. The number of systems $N$ with mass ratio less than $Q$ is plotted against $\log Q$ in full line. The simulated data for random mass selection from the Salpeter mass function with cutoff at $Q<0.1$ are plotted in dashed line

Table 5. Number of sub-systems at different levels

\begin{tabular}{l|ll}
\hline Levels & $N_{1}$ & $N_{2}$ \\
\hline $11-12$ & 519 & 164 \\
$111-112$ & 67 & 11 \\
$121-122$ & 10 & 1 \\
$1111-1112$ & 3 & 2 \\
\hline
\end{tabular}

$Q=1$ is an artifact of mass estimation procedure used in MSC.

If the masses of multiple star components were independently selected from some mass distribution and then randomly combined, one would expect to find $1 / 3$ of systems with $Q>1$, because the most massive component is more likely to belong to the pair. Surprisingly, the curve in Fig. 5 is not far from this prediction, with only slight deficiency of $Q>1$ systems. This encourages further modelling of mass distribution. The dashed curve in Fig. 5 corresponds to the simulated sample of triple stars with component masses independently chosen from the Salpeter mass function proportional to $M^{-2.35}$ in the range from 0.1 to 20 solar masses. The systems with $Q<0.1$ were excluded from the histogram. The simulation predicts $11 \%$ of such systems; presumably they are absent from the observed sample due to discovery bias. The agreement of simulated and real distributions is reasonably good and gives some support to the idea that mass ratio distribution in multiple stars may correspond to random mass combination. However, a more refined study taking into account the observational selection effects is needed.
The level asymmetry is perhaps related to maximum multiplicity of stable systems. A fully populated binary tree with 3 levels contains $2^{3}=8$ components, the systems with 4 levels also exist which can contain up to 16 components. The maximum multiplicity of a hierarchical system actually found is $7(16062-1912=\nu$ Sco $=$ HR 6027/26). Undoubtedly, the number of high-multiplicity systems and the highest known multiplicity can be increased by using modern observing techniques, but the conclusion that only a fraction of available hierarchical levels is actually filled seems to be already firmly established.

\subsection{Orbit coplanarity}

The problems of orbit coplanarity and relative sense of rotation in multiple stars has attracted much attention in the past (e.g. Fekel 1981; Worley 1967). Both problems can be treated jointly if one considers the distribution of the angle $\phi$ between angular momentum vectors of long- and short-period sub-systems. This angle can of course change due to the interaction between the orbits, but at least to the first approximation in a triple system the angular momentum of the short-period pair precesses around the total angular momentum vector which is almost parallel to the long-period momentum, so the relative angle $\phi$ must not change very much.

An attempt to use the preliminary version of MSC to probe the distribution of $\phi$ was done earlier (Tokovinin 1993) and here the results of this study are summarized. Three kinds of multiple systems were considered:

- Double-lined spectroscopic binaries belonging to visual systems with known orbit. There were 31 such systems for which the difference in orbital inclinations could be estimated. Its distribution is significantly different from what could be expected for random $\phi$ case, but strict alignment of momentum vectors is also excluded.

- Visual triple systems with 2 known orbits. Only for 3 of 14 such systems the true orientation of orbital vectors is known from radial velocity observations, for each of the remaining systems 2 values of $\phi$ are possible. The statistical arguments lead to the same conclusion, i.e. that both complete alignment and complete independence of angular momentum vectors are excluded.

- Visual binaries with known orbit and a distant physical companion. The relative sense of apparent rotation of close and distant components gives information on the average value of $\phi$ which is found to be $50^{\circ} \pm 10^{\circ}$ for 54 systems, i.e. significantly different from either $0^{\circ}$ or $90^{\circ}$.

Thus all three different samples behave in a similar way with respect to relative angular momentum orientation. The $\phi$ statistics corresponds to loosely aligned vectors, or, alternatively, to an equal mixture of well aligned and randomly aligned systems. This result has evident bearing to the formation history and dynamics of multiple systems. 


\section{Conclusions}

The multiple star catalogue is an attempt to unite most of available observational data on multiple stars. Its study leads to the following conclusions:

- MSC is complete to the distance of $10 \mathrm{pc}$ from the Sun. Only $1 / 3$ of multiple systems within 22.5 pc are actually catalogued.

- The vast majority of multiple systems are hierarchical.

- Comparison of the orbital periods of wide and close sub-systems at adjacent hierarchical levels shows that there is no preferred period ratio.

- The distribution of short periods has a depression in the range from $5 \mathrm{~d}$ to $30 \mathrm{y}$ which is likely to be due to observational selection.

- The idea that component masses are independently selected from the Salpeter mass function seems to correspond to the observed mass ratio distribution.

- Only a fraction of available hierarchical levels is filled.

- Orbital angular momentum vectors of wide and close sub-systems show a weak tendency of alignment.

MSC can serve as a database for the preparation of future observing programs concerning multiple stars. Some possible programs can be mentioned:

- Multiplicity search among nearby stars.

- Component census and the definition of the sample of complete multiple systems. Such a sample is needed for statistical study of the degree of multiplicity and level, period and mass ratio distributions. Complete component census is possible for nearby stars by combining precise radial velocity measurements over long time span with high resolution and high dynamic range imaging.

- Orbit coplanarity studies by means of interferometric resolution of close sub-systems. It will be also interesting to compare the properties of systems with coplanar and non-coplanar orbits.

- Relation of multiple stars to other stellar groups such as clusters and associations, multiplicity - age relation.

Acknowledgements. A large part of this project was accomplished while the author was a visitor at the Observatoire de Grenoble. The discussions with C. Perrier, A. Chalabaev, T.Forveille and other colleagues are gratefully acknowledged, as well as their help. F. Fekel kindly read the manuscript and suggested some improvements. The comments of the referee J. Dommanget were useful for improving the article. The extensive use of the SIMBAD database operated by the Strasbourg
University was critical for the catalogue compilation. This work was supported by the International Science Foundation (grant MPB 000) and by the France-Russia international collaboration program (PICS).

\section{References}

Abt H.A., 1988, ApJ 331, 922

Aitken R.G., 1932, New General Catalogue of Double Stars. Carnegie Inst. No. 417

Allen C.W., 1973, Astrophys. Quant. Athlone, London

Anosova J.P., 1988, Bull. Astron. Obs. Beograd 138, 13

Batten A.H., 1973, Binary and multiple systems of stars. Pergamon, Oxford

Batten A.H., Fletcher J.M., Mac Carthy D.G., 1989, Publ. DAO 17

Chambliss C.R., 1992, PASP 104, 663

Dommanget J., 1977, Rev. Mex. Astron. Astrofis. 3, 67

Dommanget J., 1988, ASS 142, 23

Duquennoy A., Mayor M., 1986, in: Hearnshaw J.B. and Cottrell P.L. (eds.) Instruments and research programs for small telescopes. Reidel, p. 59

Duquennoy, A., Mayor M., 1991, A\&A 248, 485

Evans D.S., 1968, Quart. J. R.A.S. 9, 388

Fekel F.C., 1981, ApJ 246, 879

Gliese W.H., 1969, Veröff. Astron. Rechnen Inst. Heidelberg No. 22

Gliese W.H., Jahreiss H., 1991, Preliminary Version of the Third Catalogue of Nearby Stars. NASA CD-ROM

Hoffleit D., Warren W.H., 1991, Preliminary Version of the Bright Star Catalogue, 5th Revised Edition. NASA CDROM

Larson R.B., 1995, MN 272, 213

McAlister H.A., Hartkopf W.I., 1988, Second Catalog of interferometric measurements of binary stars. CHARA Contrib. N.2, Georgia State Univ., Atlanta

Popovic G.P., 1991, Bull. Astron. Obs. Beograd 144, 13

Poveda A., Herrera M.A., Allen C., et al., 1994, Rev. Mex. Astron. Astrofis. 28, 43

Ressell H.N., Moore C.E., 1940, Masses of Stars. Univ. of Chicago

Shatskii N.I., 1996, PAZh (submitted)

Tokovinin A.A., 1992, A\&A 256, 121

Tokovinin A.A., 1993, PAZh 19, 944 (SvAL 19, 383)

Volchkov A.A., Kuz'min A.V., Nesterov V.V., 1992, in: Gulyaev A.P., Nesterov V.V. (eds.) About the four-million catalogue. Moscow Univ. Press, Moscow, p. 671

Weinberg M.D., Wasserman I., 1988, ApJ 329, 253

Worley C., 1967, Comm. Roy. Obs. Belg. Ser. B. No. 17, 221

Worley C.E., Douglass G., 1984, The Washington Double Star Catalog. U.S. Naval Obs.

Worley C.E., Heintz W.D., 1983, Publ. U.S. Naval Obs. Sec. Ser. 14 , Pt. 7 